$(p<0.05)$ were seen for knowledge and practice scores on home management of fever mainly with educational level. Multiple sources were used by the respondents for obtaining information.

Conclusions and Recommendations Gaps in the knowledge and practices of primary caregivers on childhood fever and home management of a febrile child were identified together with their socio-economic predictors.

\section{P386 NEUROLOGICAL SIGNS IN CHILDREN WITH INFLUENZA - THREE SEASONS OBSERVATIONS}

1,2Edyta Zawłocka*, 1,2Małgorzata Czajkowska, 1,22 August Wrotek, 1,2Teresa Jackowska. ${ }^{1}$ Department of Pediatrics, the Medical Centre of Postgraduate Education, Marymoncka 99/ 103, Warsaw, Poland; ' 2 Department of Pediatrics, Bielanski Hospital, Cegłowska 80, , Warsaw, Poland

\subsection{6/archdischild-2019-epa.732}

Background Influenza is an infectious disease, caused by RNA virus. Mainly, manifestations of influenza are related with the respiratory tract, but influenza can affect any other system or organ, including nervous system. The aim of the study was to analyze the frequency of neurological signs and the disease course in patients with influenza.

Material and methods 294 children aged from 12 days old, to 17 years 3 months (median 27 months) were hospitalized due to influenza during three consecutive influenza seasons (between September 2015 and August 2018). There were 197 cases of influenza type A, 76 cases of influenza type B, and 21 cases of mixed influenza $A$ and $B$ infection. Influenza was confirmed with Rapid Influenza Diagnostic Tests (RIDT) and/ or quick Real Time-Polymerase Chains Reaction (qRT-PCR).

Results $13.9 \%$ children (41 out of 294) showed neurological symptoms. The most common neurological signs were seizures $(68.3 \% ; 28 / 41)$, loss of consciousness $(21.9 \% ; 9 / 41)$ and other consciousness' disorders, and hallucinations $(9.8 \%, 4 / 41)$. In 4 patients neurological symptoms occurred without relationship with fever. $43.9 \%$ (18/41) of patients have been chronically ill, including history of feverish convulsions in three patients; one was treated because of epilepsy. No one had been vaccinated against influenza. Complications were diagnosed in $36.6 \%(15 / 41)$ patients, and antibiotic therapy was used in $46.3 \%$ of patients $(19 / 41)$.

Conclusions Influenza manifestations may be including nervous system. It should also be remembered that neurological signs and symptoms may be the first manifestation of influenza.

This study was supported by CMKP. Grant number 501-120-19-18

\section{P387 CLOSTRIDIUM DIFFICILE SEVERE INFECTION IN A NEWBORN}

${ }^{1}$ Valeria Cama, 'Luisa Pieragostini*, ${ }^{1}$ Giovanna Fontanelli, ${ }^{1}$ Maria Rosa Velletri, ${ }^{2}$ Calafiore Mariarosa. ${ }^{1}$ Great Metropolitan Hospital, Reggio Calabria , Italy; ${ }^{2}$ Great Metropolitan Hospital Melacrino Morelli, Reggio Calabria, Italy

10.1136/archdischild-2019-epa.733

Clostridium difficile infection is a rare event in newborn. Since several studies show a high rate of asymptomatic neonatal colonization by non-toxigenic Clostridium strains, C. difficile is usually not searched in infants and children affected by bloody diarrhea and younger than 12 months. Nevertheless, Clostridium difficile infection should be suspected in patients with bloody diarrhea and other comorbidities. The long term hospitalization and the prolonged exposure to broad-spectrum antibiotic therapy, are also important risk factors for intestinal infection. We present the case of a 10-day newborn with thrombocytopenia associated with rectorrhagia and positivity of inflammation indices. Transfusion of platelets and immunoglobulins was performed with platelet normalization and empiric antibiotic therapy was practiced (ampicillin and gentamicin). A sudden clinical worsening occurred after ten day: he developed severe bloody diarrhea with systemic failure. Laboratory tests showed: neutrophilic leukocytosis, thrombocytosis, anemia, an important increase in inflammation indices. Several abdomen X-ray showed moderate gaseous distension of some intestinal loops with presence of hydroaerial levels. Abdominal ultrasound revealed diffuse thickening of the colon walls (a sign of colitis). The search of C. difficile in stools sample was positive for C. difficile producer of Toxin $A$ and toxin B Enzym Immunoassay (EIA). The infant was successfully treated with vancomycin and metronidazole, without relapsing.

\section{P388 RSV AND INFLUENZA CO-INFECTIONS IN CHILDREN IN THREE CONSECUTIVE INFLUENZA EPIDEMIC SEASONS}

1,2Edyta Zawłocka*, 1,2 Małgorzata Czajkowska, ${ }^{1,2}$ August Wrotek, ${ }^{1,2}$ Teresa Jackowska. ${ }^{1}$ Department of Pediatrics, the Medical Centre of Postgraduate Education, Marymoncka 99/ 103, Warsaw, Poland; 'Department of Pediatrics, Bielanski Hospital, Cegłowska, Warsaw, Poland

\subsection{6/archdischild-2019-epa.734}

Background Influenza and Respiratory Syncytial Virus (RSV) infections are severe diseases in children, especially in neonates and young infants. Lot of them often lead to hospitalization.

There are no reports on the frequency of RSV and influenza co-infections.

Material and methods In last three influenza epidemic seasons - from September 2015 to August 2018 we diagnosed 301 cases of influenza. 294 of our patients were hospitalized. 15 patients (4.1\%) were diagnosed RSV-influenza co-infection. Majority of the group was male $(55.4 \%$ vs. $44.6 \%)$, opposite to the co-infection group (12 girls and 3 boys).

Results 12 (80\%) with RSV infection were infected with influenza type A, 3- influenza B and 2 patients with type A and B. $86.7 \%(13 / 15)$ patients with co-infection were at the age of 6 months or less. 12 patients $(80 \%)$ obtained antibiotics, while in control group it was administered in $36.1 \%$ of cases. Frequency of complications in co-infected group was 93\%, while $51.7 \%$ of patients in control group had complications. 1 patient was transferred to the intensive care unit (ICU). Relative risk (RR) of complications was 1.8.

Conclusion We observed high incidence of RSV-influenza coinfections, which were related especially, to influenza type A virus. It confirms that one pathogen may co-exist with the other. The course of the disease in patients with RSV and influenza co-infection is usually severe burdened with high risk of complications.

This study was supported by CMKP. Grant number 501-120-19-18 